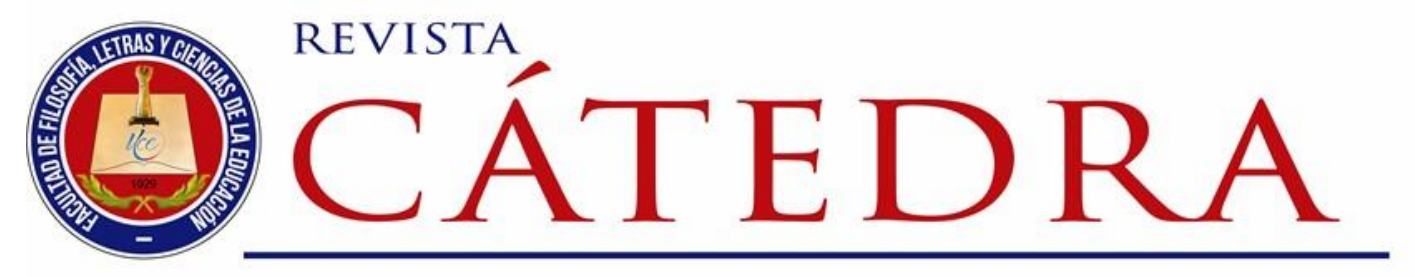

\title{
Relación entre rendimiento académico y asistencia como factores de promoción estudiantil
}

\section{Relation between academic performance and attendance as factors of student promotion}

\author{
Segundo Barreno-Freire \\ Universidad Central del Ecuador, Quito, Ecuador \\ sbarreno@uce.edu.ec \\ https://orcid.org/0000-0003-0845-5360 \\ Oswaldo Haro-Jácome \\ Universidad Central del Ecuador, Quito, Ecuador \\ oharo@uce.edu.ec \\ https://orcid.org/0000-0001-6387-9591 \\ Paola Flores-Yandún \\ Universidad de Guayaquil, Quito, Ecuador \\ paola.floresy@ug.edu.ec \\ https://orcid.org/0000-0002-9861-4347
}

(Recibido: 13/10/2018; Aceptado: 20/11/2018; Versión final recibida: 13/12/2018)

Cita del artículo: Barreno-Freire, S., Haro-Jácome, O. y Freire-Yandún, P. (2019). Relación entre rendimiento académico y asistencia como factores de promoción estudiantil. Revista Cátedra, 2(1), 44-59.

\section{Resumen}

Este artículo aborda la correspondencia entre el rendimiento académico y la asistencia como factores de promoción del estudiantado de la Facultad de Filosofía, Letras y Ciencias de la Educación (FFLCE) de la Universidad Central del Ecuador (UCE). La información documental que sirvió de base para la investigación fue extraída de los archivos institucionales que registró la Unidad de Servicios Informáticos de la Facultad de Filosofía (USIF) en el período académico 2016 - 2017. El componente principal que se analizó en el 
sistema fue el relacionado con la variable calificaciones de todos los niveles (semestres), considerados los promedios de las asignaturas de las diez carreras vigentes para titulación, aprobadas por el Consejo de Educación Superior (CES), en las que constan matriculados 3617 estudiantes. Los promedios de las calificaciones y asistencias fueron calculados mediante un análisis estadístico con el uso del coeficiente de correlación y se obtuvo un 0.75 , lo cual significa que hay una correlación positiva moderada y directamente proporcional. El rendimiento académico y asistencia por la variable sexo del estudiantado (hombres - mujeres) no evidencia diferencia significativa, lo que quiere decir que los estudiantes independientes del sexo tienen similares calificaciones y asisten por igual a las clases. Es de vital importancia el estudio, pues con base en los resultados que se muestran se pueden tomar decisiones en relación a las carreras que presentan menor promedio como en el caso de Informática.

\section{Palabras clave}

Aprobación, asistencia, calificaciones, promedios, promoción estudiantil, rendimiento académico, reprobación.

\section{Abstract}

This article shows the correspondence between academic performance and attendance as factors of success of the students studying at the Faculty of Philosophy and Education Sciences (FFLCE) of Universidad Central del Ecuador (UCE), from documentary information of the institutional files registered by the Computer Services Unit of the Faculty of Philosophy (USIF) in the academic period 2016 - 2017, specially the qualifications component of all the levels (semesters), considered the grade-point averages of the subjects about current careers for the degree, approved by the Council of Higher Education (CES), in which 3617 students are enrolled. The grade-point averages and attendance were calculated through a statistical analysis with the use of the correlation coefficient, and a 0.75 was obtained. This means that there is a moderate positive correlation, and directly proportional. Academic performance and attendance by gender (men - women) does not show significant difference, which means that they have similar qualifications and attend to classes equally.

\section{Keywords}

Academic performance, grades, attendance, student promotion.

\section{Introducción}

Las funciones fundamentales de la universidad son docencia, investigación y vinculación con la sociedad como un todo dinámico e integral cuyo fin último es la formación profesional, la producción científica y el aporte a la solución de problemas de la sociedad. De las tres funciones, la docencia muestra ser prioritaria en la gestión universitaria, ya que procura profesionalizar a la juventud con sólidos conocimientos científicos, técnicos y humanísticos para que aporten al desarrollo de la sociedad ecuatoriana.

El sistema educativo ecuatoriano reconoce todos los niveles de formación estudiantil. Considera el nivel superior como prioritario, pues es el factor de promoción denominado rendimiento académico, al cual se lo conceptualiza: 
[...] en ocasiones se le denomina como aptitud escolar, desempeño académico o rendimiento escolar, pero generalmente las diferencias de concepto sólo se explican por cuestiones semánticas, ya que generalmente, en los textos, la vida escolar y la experiencia docente, son utilizadas como sinónimos (Navarro, 2003, pág. 2).

El rendimiento académico determina la aprobación o reprobación de un semestre, un curso o un módulo, así como el nivel de éxito terminal luego de una etapa formativa como el grado o semestre. La categoría rendimiento académico, ha sido investigada a nivel nacional y mundial, llegando a establecer diversas conceptualizaciones en el ámbito educativo, que se configura como multicausal, porque engloba una serie de aspectos que intervienen en el proceso y constituye el principal indicador de logros de aprendizaje. Es precisamente esta categoría conceptual que se aborda en el artículo, desde una perspectiva cuantitativa, fundamentado en el análisis estadístico. Quizás la definición más acertada es:

que el rendimiento académico es la suma de diferentes y complejos factores que actúan en la persona que aprende. Ha sido definido con un valor atribuido al logro del estudiante en las tareas académicas. Se mide mediante las calificaciones obtenidas, con una valoración cuantitativa, cuyos resultados muestran las materias ganadas o perdidas, la deserción y el grado de éxito académico (Garbanzo, 2007, pág.46).

Las investigaciones desarrolladas sobre esta compleja temática concuerdan que para lograr un buen rendimiento académico deben conjugarse integralmente algunas importantes dimensiones. Los factores o dimensiones fundamentales que impactan claramente en el rendimiento académico son: la dedicación y el esfuerzo al aprendizaje, la habilidad académica, el contexto socioeconómico, la motivación, los estilos de aprendizaje y enseñanza; entre otros factores que influyen de manera directa en las calificaciones obtenidas por los estudiantes. En otras palabras, a través del rendimiento académico se determina logros de aprendizaje alcanzado por el estudiante y se expresa mediante una calificación sea cualitativa o cuantitativa, o con sus equivalencias cuanti-cualitativas, producto de la valoración de un conjunto de actividades académicas planificadas para el efecto.

El tratado aborda las relaciones que tienen en el rendimiento académico con la asistencia a clases y la diferencia entre la variable sexo de los estudiantes (hombre y mujer). Las reflexiones planteadas en el presente estudio, buscan exponer y explicar el rendimiento académico alcanzado por los y las estudiantes de las diez carreras vigentes en la FFLCE de la Universidad Central del Ecuador (UCE). Los promedios de calificaciones y asistencia que los estudiantes tienen por asignatura en el semestre 2016-2017, fueron elementos considerados en el análisis.

El Instructivo de Evaluación de la UCE, con su correspondiente Sistema Académico, con base en el Reglamento de Régimen Académico 2016 del Consejo de Educación Superior (CES), tiene establecido que en el semestre cada estudiante obtiene dos calificaciones hemisemestrales sobre 20 puntos cada una. Estas calificaciones deben sumar como mínimo 28/40 puntos para aprobar cada asignatura. Los estudiantes que tienen entre 8 y menos de 28 puntos, tienen derecho al examen de recuperación cuya nota (sobre 20) sumada al promedio logrado en los dos hemisemestres deberá ser de 28 puntos por lo menos para aprobación, y si tienen menos de 8 puntos, automáticamente están reprobados.

Licencia Creative Commons Atribución 4.0 Internacional (CC BY 4.0)

Revista Cátedra, 2(1), pp. 44-59, enero-abril 2019. e-ISSN: 2631-2875

https://doi.org/10.29166/catedra.v2i1.1552 
El propósito de la investigación es analizar la relación que se manifiesta entre el rendimiento académico con la asistencia a clases, considerando la variable sexo de los estudiantes matriculados en la Facultad de Filosofía en el periodo 2016-2017. Las carreras pertenecientes a la Facultad son: Ciencias del Lenguaje y Literatura, Ciencias Naturales, Ciencias Sociales, Comercio y Administración, Informática, Inglés, Matemática y Física, Parvularia, Plurilingüe, Psicología Educativa, las mismas que en el semestre de la investigación están en proceso de aprobación de sus rediseños y diseños curriculares, y que a partir del período siguiente cambiaran su nomenclatura. Las carreras tienen, entre 49 y 69 asignaturas distribuidas en los nueve semestres para la Licenciatura en Ciencias de la Educación. Los datos analizados son las notas de los estudiantes obtenidos por asignatura en cada una de las carreras, de lo cual se sistematizó el promedio de las calificaciones por estudiante y por carrera.

La segunda variable analizada fue la asistencia de los estudiantes a clases, las mismas que se obtuvo de la base de datos de la Facultad en valores porcentuales, de la que se calculó el promedio por carrera, considerando los promedios de asistencia que presentan los estudiantes por cada asignatura. La normativa de la UCE sobre asistencia señala que para aprobar una asignatura el o la estudiante debe lograr el estándar mínimo del 80\% durante el semestre (Rodríguez, 2016). La población estudiantil de la Facultad matriculada en el período académico 2016 - 2017 es de 3617 estudiantes, de los cuales, clasificando por sexo: el $35,44 \%$ son hombres y el $64,56 \%$ son mujeres; distribuidos en las diez carreras y en los nueve semestres académicos que corresponde a la formación profesional de grado.

El estudio busca determinar si la calidad de asistencia a clases mejora el rendimiento académico estudiantil. Además, establecer si hay diferencias entre el rendimiento académico de estudiantes hombres y mujeres; para lo cual se presenta resultados estadísticos mediante cuadros y gráficos que ilustran las características de las variables de investigación. Al final del estudio se pretende identificar si existe relación entre el porcentaje de la asistencia estudiantil con los promedios de las calificaciones del estudiantado. También se hace una comparación de los promedios de las calificaciones, los porcentajes de asistencia entre estudiantes hombres y mujeres por carreras y en el conjunto de la Facultad.

El artículo está estructurado en cinco secciones: introducción; fundamento teórico de las variables de estudio en relación a la conceptualización de rendimiento académico; calificaciones; asistencia y promoción estudiantil. Además, se profundiza en la metodología empleada en el estudio, los resultados alcanzados, discusión, conclusiones y bibliografía.

\section{Rendimiento académico}

El rendimiento académico estudiantil es el aspecto más trascendente de todo proceso educativo, si se trata de observar calidad educativa porque para el: “(...) estudiantado universitario constituye un factor imprescindible en el abordaje del tema de la calidad de la educación superior, debido a que es un indicador que permite una aproximación a la realidad educativa" (Garbanzo, 2007, pág. 43). La enseñanza se constituye por acciones planificadas y desarrolladas fundamentalmente por los docentes para lograr un resultado de aprendizaje en el estudiante como un esfuerzo intelectual asumido, y que se concreta en el rendimiento académico observado a través de la evaluación sumativa, por lo que, sobre el concepto rendimiento plantea que: 
Resulta evidente, que el abordaje del rendimiento académico no podría agotarse a través del estudio de las percepciones de los alumnos sobre las variables habilidad y esfuerzo, así como tampoco podría ser reducida a la simple comprensión entre actitud y aptitud del estudiante. La demanda de análisis y evaluación de otros factores permiten infiltrarnos más en el rendimiento académico como fenómeno de estudio, [...] se abordarán variables, que van desde su conceptualización, predicción y evaluación hasta la investigación desarrollada en diferentes niveles educativos, [...]. (Navarro, 2003, pág. 2).

El rendimiento académico involucra una serie de componentes como la habilidad, el esfuerzo, la actitud y la aptitud del estudiante. Además, se puede explicar al rendimiento académico como el resultado de todo el proceso de enseñanza y aprendizaje, implementado de manera regular y no regular, obtenido de forma individual y colectiva a través de la evaluación.

El rendimiento académico como producto final de un proceso evaluativo está mediado por factores sociales del contexto, sin embargo, también coexisten factores motivacionales intrínsecos y extrínsecos que favorecen o dificultan el aprendizaje. La presencia de factores pedagógicos, didácticos y curriculares también determinan el rendimiento y hacen visibles los métodos didácticos, recursos, destrezas, contenidos y valores que se usan para dicho proceso evaluativo. La evaluación es por ende parte fundamental en el proceso enseñanzaaprendizaje. Al respecto, Arribas (2014) señala: "una de las manifestaciones principales del rendimiento académico son las calificaciones" (pág.2).

En este artículo, se hace referencia a un elemento del rendimiento académico como son las calificaciones, obtenidas a través de la evaluación del proceso de aprendizaje en sus etapas finales para acreditación en un sistema o subsistema educativo. Si bien es cierto, las calificaciones entrañan una dosis alta de subjetivismo y en muchos casos no reflejan las capacidades reales logradas por los estudiantes, sin embargo, son los resultados más evidentes del rendimiento académico alcanzado por ellos.

\subsection{Calificaciones y rendimiento académico}

Es necesario también considerar una perspectiva distinta sobre el aprendizaje, en el que hay mayor determinación del proceso frente al producto o rendimiento. Al respecto, Carretero (2009) indica:

[...] aprender es sinónimo de comprender. Por ello, lo que se comprende es lo que se aprende y lo que luego se recordará mejor, porque queda integrado en nuestra estructura de conocimientos. Por tanto, resulta fundamental para el profesor no sólo conocer las representaciones que poseen los alumnos sobre lo que se les va a enseñar, sino también analizar el proceso de interacción entre el conocimiento nuevo y el que ya poseen. De esta manera, no es tan importante el producto final que emite el alumno como el proceso que lo lleva a dar una determinada respuesta (pág. 2).

En el ámbito de la academia, existe una controversia muy fuerte en torno a señalar a las notas o calificaciones como la expresión más evidente del rendimiento académico. Es sustancial tener conciencia de que existen otros aspectos también importantes almomento

\section{Licencia Creative Commons Atribución 4.0 Internacional (CC BY 4.0)}

Revista Cátedra, 2(1), pp. 44-59, enero-abril 2019. e-ISSN: 2631-2875

https://doi.org/10.29166/catedra.v2i1.1552 
de analizar los aprendizajes generados en un proceso educativo como son: las destrezas, habilidades, conocimientos, comportamientos, práctica de valores, pertinencia social, entre otros. Sin embargo, las calificaciones son los indicadores primordiales para determinar el rendimiento académico, el mismo que se expresa con una valoración cualitativa o cuantitativa que obtiene el estudiante.

De acuerdo a Murillo (2013), contextualiza al rendimiento académico como la suma de un conjunto de elementos variados, complejos e interdependientes que participan en todo proceso orientado al aprendizaje. El mismo que es evaluado de manera permanente y como resultado se obtiene las calificaciones a través de una escala generalmente cuantitativa con su correspondiente equivalencia cualitativa, que evidencian aprobación o reprobación en cada asignatura (Murillo, 2013, págs. 120-121). De igual manera, Garbanzo (2007) asume una conceptualización similar a la expuesta anteriormente, que en esencia sostiene lo siguiente:

La valoración del rendimiento académico no conduce a otra cosa que a la relación entre lo que se aprende y lo que se logra desde el punto de vista del aprendizaje, y se valora con una nota, cuyo resultado se desprende de la sumatoria de la nota de aprovechamiento del estudiante en las diferentes actividades académicas a las que se sometió en un ciclo determinado (pág. 46).

En este contexto, el resultado de las evaluaciones, expresadas en calificaciones de manera cualitativa o cuantitativa, son en efecto las evidencias más empleadas para verificar resultados cuando se estudia al rendimiento académico. El Reglamento del Sistema de Evaluación Estudiantil RSEE (2017), aprobado por el Consejo de Educación Superior CES, implementa lineamientos generales para desarrollar la evaluación de los aprendizajes. Además, establece que cada Universidad debe elaborar un sistema interno de evaluación, con especificidades propias de la institución, obviamente contemplando las directrices emanadas por el CES. En el mencionado reglamento se conceptualiza a la evaluación de los aprendizajes en los siguientes términos:

Artículo 5.- La evaluación como componente del aprendizaje.- La evaluación de los aprendizajes constituye un pilar fundamental dentro del proceso educativo de los estudiantes, de las carreras y programas, que siendo sistemático, permanente y participativo permite la valoración integral de sus avances en la adquisición de capacidades cognitivas, investigativas, procedimentales y actitudinales, de tal forma que contribuyan a garantizar la calidad e integralidad de la formación profesional (CES, 2016, pág. 4).

Para la evaluación se han establecido periodos académicosdivididos en dos hemisemestres. Cada hemisemestre se aplica un proceso de evaluación que consiste en un examen que tiene un peso del $40 \%$ de la calificación. En el proceso evaluativo también se consideran otras estrategias como: trabajos en equipo, trabajo autónomo, investigaciones, proyectos entre otros, esto corresponde al $60 \%$ de los 20 puntos.

La evaluación de los aprendizajes se expresa en resultados considerando las siguientes escalas; calificaciones cualitativas: excelente, muy bueno, bueno, regular y deficiente. Mientras que las calificaciones cuantitativas se establecen en una escala de 0 a 20 puntos. 
Para la aprobación de cada asignatura, el estudiantado, debe obtener al menos 28 puntos en la sumatoria de notas de los dos hemisemestres. En caso de no alcanzar este puntaje, el estudiante tiene derecho a una evaluación adicional de recuperación (supletorio), siempre que obtenga una calificación superior a 8,8 puntos en el semestre; si la calificación es menor, reprueba la asignatura. La evaluación de recuperación está compuesta por el $60 \%$ de la calificación que obtenga en el examen de recuperación, más el $40 \%$ de la suma de las calificaciones obtenidas en el primero y segundo hemisemestre. De igual forma para la aprobación de una asignatura el estudiante debe alcanzar como mínimo una calificación de 28 puntos en la evaluación de recuperación, caso contrario reprueba la asignatura. El estándar mínimo para promoción semestral por asignatura en la UCE es de 28/40 puntos.

\subsection{Asistencia}

En el ámbito de la educación superior la asistencia a clases regulares es definida “(...) como la asistencia diaria del alumno a las clases" (Universidad de las Palmas Gran Canaria, 2011, p. 1), la misma que es obligatoria en la modalidad presencial, por lo que el estudiante personalmente debe llegar puntualmente a tomar participación en cada una en las diferentes asignaturas en las que se haya matriculado en el respectivo período académico. Cada docente, por su parte, es responsable de registrar las asistencias de los estudiantes, individualmente, en cada hora de trabajo de aula, y al finalizar cada hemisemestre debe consignar el total de asistencias al sistema informático del a UCE. La Universidad de Loyola - Andalucía, en la guía docente manifiesta que:

La asistencia a clase es un elemento esencial dentro del proceso de aprendizaje de la asignatura, junto con la participación activa del alumno en su desarrollo y el trabajo continuo. La configuración de la asignatura necesita la asistencia del alumno y la participación en clase para que el alumno pueda alcanzar los objetivos y competencias de la asignatura (Universidad de Loyola Andalucía, s.f., portada).

La asistencia se refiere a la presencia física del estudiante en las clases de las diferentes asignaturas, esto, en la modalidad de estudios presenciales. Para las Instituciones de Educación Superior (IES) del Ecuador, según el Reglamento de Régimen Académico promulgado por el CES el año 2013, en su reforma de marzo de 2017, consta que las licenciaturas deben cumplir con 7200 horas. Estas horas deben estar distribuidas en 54 asignaturas, organizadas en tres unidades curriculares: básica, profesional y titulación, durante los nueve periodos académicos que duran estas carreras de tercer nivel. Este número de horas contempla el trabajo presencial asistido por el docente más el trabajo autónomo desarrollado por el estudiante en un periodo semestral de 16 semanas (CES, 2017).

Para la aprobación de una asignatura, estudiantes y docentes cumplen la normativa legal vigente de ámbito nacional e institucional, en lo relacionado con la evaluación de los aprendizajes en las que se integran dos componentes: la calificación y la asistencia obtenida por parte del estudiante en el semestre. De acuerdo al instructivo para la evaluación estudiantil de grado emitido por la UCE en mayo de 2017, en el numeral 12 se dispone que: "Las asignaturas, cursos o equivalentes que deban tomar los estudiantes dentro de su etapa de formación serán aprobados con un mínimo del 70\% de la calificación y una asistencia mínima del 80\% de clases presenciales" (UCE, 2017, p. 3). Con este mandato coincide la disposición de Vicerrectorado Académico de la UCE de octubre de 2016, que sirvió de base para analizar la asistencia estudiantil en el presente trabajo académico. 


\subsection{Promoción estudiantil}

La promoción estudiantil en la universidad es entendida como la aprobación de un curso, materia o módulo luego de un tiempo reglamentario de estudios y esfuerzo personal, como define (Suárez, 2011) "[...] el paso de un curso a otro cuando los objetivos han sido superados por el alumno tanto educativos como personales" (pág. 1). Para que suceda la aprobación, es decir, el paso a una situación nueva y superior, son necesarios varios requisitos constantes en las normativas generales y particulares de las IES. En el caso de la UCE, para la promoción de nivel escolar, el estatuto universitario disponeque:

Art. 160.- Aprobación de cursos y asignaturas. - las asignaturas o cursos que deban tomar los estudiantes dentro de su etapa de formación serán aprobadas con un mínimo del $70 \%$ de la calificación y una asistencia mínima del 80\% de clases presenciales (UCE, 2016, pág. 70).

En relación a lo anteriormente citado, la UCE en todas sus carreras de grado dispone como dos requisitos obligatorios para promocionar a sus estudiantes: las calificaciones y asistencias por asignaturas y semestres. La calificación y asistencia son determinantes, pues al no cumplir con el porcentaje asignado a cada una de ellas, el estudiante no será promovido al nivel superior.

\section{Metodología}

El estudio fue de tipo no experimental, transversal y descriptivo, en razón que únicamente se describen las variables en observación, sin intervenir con tratamiento; los datos corresponden a un periodo académico; la unidad de análisis son los resultados de las calificaciones estudiantiles del semestre 2016-2017. Los datos para el análisis descriptivo fueron extraídos del archivo informático de la Facultad de Filosofía de la UCE, a cargo de la Unidad de Servicios Tecnológicos de la Facultad de Filosofía (USIF). El estudio también es abordado desde la investigación documental, con la revisión de argumentos planteados por varios autores. La información y contenidos seleccionados fueron trascendentes para el estudio. A esta selección de contenidos se integró datos empíricos de calificaciones y asistencias que facilitó la USIF, los mismos que fueron sometidos a una reflexión profunda para establecer conclusiones válidas respecto al estudio del tema.

La población inmersa en la investigación es: $\mathrm{N}=3617$ estudiantes matriculados en el semestre 2016-2017 en las diez carreras de la Facultad de Filosofía, Letras y Ciencias de la Educación como se muestra en el Cuadro 1. La población está dividida en hombres y mujeres con la finalidad de correlacionar la variable sexo con el rendimiento académico y asistencia.

\begin{tabular}{lrrr}
\hline \multirow{2}{*}{ Carreras } & Muieres & \multicolumn{3}{c}{ Población estudiantil } \\
Hombres & Total \\
\hline Ciencias del Lenguaje y Literatura & 212 & 119 & 331 \\
Ciencias Naturales & 225 & 139 & 364 \\
Ciencias Sociales & 200 & 174 & 374 \\
Comercio y Administración & 82 & 48 & 130 \\
Informática & 71 & 250 & 321 \\
Inglés & 209 & 128 & 337 \\
Matemática y Física & 113 & 173 & 286 \\
Parvularia & 498 & 3 & 501 \\
Plurilingüe & 240 & 77 & 317 \\
\hline
\end{tabular}




\begin{tabular}{lrrr}
\hline Psicología Educativa & 485 & 171 & 656 \\
TOTAL FFLCE & 2335 & 1282 & 3617 \\
& $(64,56 \%)$ & $(35,44 \%)$ & $(100 \%)$ \\
\hline
\end{tabular}

Cuadro 1. Población Estudiantil Facultad de Filosofía, Letras y Ciencias de la Educación (FFLCE)

Como consta en el cuadro 1 la mayoría de la población es de sexo femenino, esto es el $64,56 \%$, en tanto que los hombres son el $35,44 \%$, lo que significa que la Facultad tienen una composición mayoritaria de población femenina en el período estudiado. Además, en carreras como Parvularia, Psicología y Plurilingüe que son las más grandes, tienen más del 80\% estudiantes mujeres. Las carreras investigadas están en proceso de aprobación de sus rediseños y diseños curriculares y consecuentemente cambiarán su nomenclatura a partir del siguiente período académico.

\section{Resultados y discusión}

Los resultados que se presentan a continuación, son provenientes del análisis estadístico realizado con los datos correspondientes a las variables de estudio. Así se presenta la asistencia expresada en porcentaje sobre $100 \%$ y el rendimiento académico en escala de calificaciones semestrales de 0 a 40 puntos que corresponde al semestre 2016-2017.

\begin{tabular}{lrrr}
\hline Carreras & $\begin{array}{l}\text { Promedio } \\
\text { calificaciones }\end{array}$ & $\begin{array}{l}\text { Promedio } \\
\text { asistencia }\end{array}$ & $\begin{array}{l}\text { Coeficiente } \\
\text { correlación }\end{array}$ \\
\hline Ciencias del Lenguaje y Literatura & 33,43 & $92,73 \%$ & 0,80 \\
Ciencias Naturales & 32,16 & $93,05 \%$ & 0,76 \\
Ciencias Sociales & 31,90 & $94,70 \%$ & 0,48 \\
Comercio y Administración & 29,25 & $86,82 \%$ & 0,77 \\
Informática & 28,72 & $88,37 \%$ & 0,75 \\
Inglés & 32,57 & $94,78 \%$ & 0,48 \\
Matemática y Física & 30,04 & $93,86 \%$ & 0,57 \\
Parvularia & 33,99 & $94,78 \%$ & 0,66 \\
Plurilingüe & 32,08 & $91,22 \%$ & 0,81 \\
Psicología Educativa & 32,80 & $94,14 \%$ & 0,70 \\
TOTAL FFLCE & 31,69 & $92,45 \%$ & 0,75 \\
\hline
\end{tabular}

Cuadro 2. Promedio de calificaciones y asistencias por carrera en la FFLCE

Los promedios de calificaciones por carrera, se muestra en el cuadro 2, y se expresa en una escala sobre 40 puntos, siendo la carrera de Parvularia la que tiene el promedio más alto (33,99/40 puntos) en rendimiento académico estudiantil. El análisis también muestra que la carrera de Informática presenta el promedio más bajo (28,72/40 puntos).

El promedio de rendimiento académico considerando las 10 carreras de la Facultad es de 31,69/40 puntos, superior en 3, 69 puntos al estándar mínimo de promoción de la UCE de 28/40 puntos, pero inferior en 8,31 del estándar máximo de 40/40; por lo tanto, su nivel se valora como Bueno según (Rodríguez, 2017). Luego hay dos grupos de carreras ubicadas arriba y abajo del promedio general de rendimiento académico; 8 carreras tienen promedios superiores pero cercanos a la media. Solo dos carreras están por debajo, pero con promedios alejados de la media.

\section{Licencia Creative Commons Atribución 4.0 Internacional (CC BY 4.0)}

Revista Cátedra, 2(1), pp. 44-59, enero-abril 2019. e-ISSN: 2631-2875

https://doi.org/10.29166/catedra.v2i1.1552 
El promedio de asistencia en cambio, la media aritmética de asistencia de las 10 carreras es de 92,45\%; siendo las carreras de Parvularia e Inglés que alcanza el porcentaje mayor de asistencia $(94,78 \%)$; mientras que la carrera de Comercio tiene en promedio menor con el $86,82 \%$ de asistencia. Llama la atención que en Ciencias Sociales hay porcentajes de asistencia en pocas asignaturas con valores superiores al 100\%. Seis carreras superan a la media, mientras que cuatro tienen promedios inferiores a la media. El promedio de asistencia estudiantil de la Facultad supera en 12,45 puntos porcentuales al estándar mínimo establecido por laUCE.

\subsection{Prueba de correlación}

La prueba estadística calculada fue el coeficiente de correlación $r$ de Pearson, entre el promedio de las calificaciones y el promedio de asistencias, considerando la totalidad estudiantes en todas las asignaturas de las diez carreras. El índice de correlación en las diferentes carreras está en un intervalo de 0,48 a 0,81 y a nivel de la Facultad es de 0,75 como se muestra en la Cuadro 2.

Al hacer la interpretación del coeficiente de correlación de Pearson que es de 0,75; significa que existe una correlación positiva alta, según las equivalencias del cuadro 3 . Al ser positivo el coeficiente, significa que entre las dos variables hay una relación directamente proporcional, en otras palabras, si el promedio de las calificaciones sube, también aumenta el porcentaje de asistencias. Sin embargo, se debe señalar que en las carreas de Ciencias Sociales e Inglés la correlación es moderada entre las calificaciones y la asistencia a diferencia de las otras carreras.

\begin{tabular}{lr}
\hline$>0.80$ & Muy alta \\
$0.60-0.79$ & Alta \\
$0.40-0.59$ & Moderada \\
$0.20-0.39$ & Baja \\
$<0.20$ & Muy baja \\
\hline
\end{tabular}

Cuadro 3. Interpretación de los coeficientes de correlación

Fuente: http://personales.unican.es/salvadol/apuntes2b.pdf

\subsection{Aprobados y Reprobados}

Los estudiantes para aprobar una asignatura deben sumar como mínimo 28 puntos y además superar el $80 \%$ de asistencias a clases en cada asignatura. La tendencia de estudiantes aprobados y reprobados se presenta en el Cuadro 4.

\begin{tabular}{lrrr}
\hline Carreras & $\begin{array}{l}\text { Número de } \\
\text { estudiantes }\end{array}$ & $\begin{array}{l}\text { Porcentaje de } \\
\text { aprobados } \\
\text { Total asignaturas }\end{array}$ & $\begin{array}{l}\text { Porcentaje de } \\
\text { reprobados } \\
\text { Total asignaturas }\end{array}$ \\
\hline Ciencias del Lenguaje y Literatura & 331 & $96,29 \%$ & $3,71 \%$ \\
Ciencias Naturales & 364 & $94,53 \%$ & $5,47 \%$ \\
Ciencias Sociales & 374 & $93,56 \%$ & $6,44 \%$ \\
Comercio y Administración & 130 & $87,57 \%$ & $12,43 \%$ \\
Informática & 321 & $79,87 \%$ & $20,13 \%$ \\
Inglés & 337 & $93,59 \%$ & $6,41 \%$ \\
Matemática y Física & 286 & $86,28 \%$ & $13,72 \%$ \\
Parvularia & 501 & $97,42 \%$ & $2,58 \%$ \\
Plurilingüe & 317 & $93,02 \%$ & $6,98 \%$ \\
\hline
\end{tabular}




\begin{tabular}{lccc}
\hline & 656 & $95,19 \%$ & $4,81 \%$ \\
Psicología Educativa & & $91,73 \%$ & $8,27 \%$ \\
PROMEDIO FFLCE & 3617 & 917 \\
\hline
\end{tabular}

Cuadro 4. Porcentaje de estudiantes aprobados y reprobados por carrera en la FFLCE

El promedio de estudiantes reprobados está el 8,27\% en la Facultad, es decir que no han superado el puntaje mínimo requerido en rendimiento académico, en asistencia o en los dos requisitos. Las carreras que tienen un menor índice de estudiantes reprobados son: Parvularia con el 2,58\%; Ciencias del Lenguaje tiene el 3,71\% y Psicología con el 4,81\%. En el otro extremo, se encuentran tres carreras que presentan un mayor índice de repitencia, estas son: Informática con el 20,13\%; Matemática y Física con el 13,72\% y Comercio y Administración con el 12,43\% de reprobados. Llama la atención el índice de reprobados en estas carreras, por lo que, se requiere hacer una investigación más profunda para determinar las causas de este problema académico.

Asignaturas por carreras. Es importante señalar que las asignaturas que tienen en las Carreras varían numéricamente; existe un bloque de asignaturas comunes, que están orientadas a la formación profesional docente en todas las carreras de la Facultad; y otro bloque de asignaturas que se desprenden de la formación especializada de cada carrera. La carrera de Inglés presenta un menor número con 41 asignaturas, mientras que, la carrera de Parvularia tiene 69 asignaturas y la media de asignatura por carreras es de 56,5 en la Facultad de Filosofía. En el Cuadro 5 se presenta el número de asignaturas por carrera.

\begin{tabular}{lr}
\hline Carreras & $\begin{array}{c}\text { Número de asignaturas } \\
\text { Periodo 2016 - 2017 }\end{array}$ \\
\hline Ciencias del Lenguaje y Literatura & 56 \\
Ciencias Naturales & 58 \\
Ciencias Sociales & 53 \\
Comercio y Administración & 49 \\
Informática & 55 \\
Inglés & 41 \\
Matemática y Física & 66 \\
Parvularia & 69 \\
Plurilingüe & 59 \\
Psicología Educativa & 59 \\
MEDIA FFLCE & 56,5 \\
\hline
\end{tabular}

Cuadro 5. Número de asignaturas por carrera en la FFLCE

Diferencia entre rendimiento académico y asistencia por sexo. Se considera la variable sexo para diferenciar esencialmente las calificaciones y asistencia logradas por estudiantes hombres y mujeres. En el Cuadro 6, se hace un análisis comparativo de los valores promedio obtenidos en las notas y la asistencia a clases en todas las asignaturas por parte del estudiantado de todas las carreras.

\begin{tabular}{lcccccc}
\hline \multirow{2}{*}{ Carreras } & \multicolumn{2}{c}{ Hombres } & \multicolumn{2}{c}{ Mujeres } & \multicolumn{2}{c}{ Total } \\
& Calificaciones & Asistencias & Calificaciones & Asistencias & Calificaciones & Asistencias \\
\hline $\begin{array}{l}\text { Ciencias } \\
\text { del Lenguaje } \\
\text { y Literatura }\end{array}$ & 33,43 & $92,71 \%$ & 33,43 & $92,75 \%$ & 33,43 & $92,73 \%$ \\
& & & & & &
\end{tabular}




$\begin{array}{lllllll}\text { Ciencias Naturales } & 32,15 & 93,04 \% & 32,16 & 93,05 \% & 32,16 & 93,05 \% \\ \text { Ciencias Sociales } & 31,90 & 94,70 \% & 31,89 & 94,69 \% & 31,90 & 94,70 \% \\ \text { Comercio y } & 29,31 & 86,92 \% & 29,18 & 86,72 \% & 29,25 & 86,82 \% \\ \text { Administración } & 28,72 & 88,37 \% & 28,72 & 88,37 \% & 28,72 & 88,37 \% \\ \text { Informática } & 32,57 & 94,78 \% & 32,56 & 94,77 \% & 32,57 & 94,78 \% \\ \text { Inglés } & 30,04 & 93,87 \% & 30,03 & 93,84 \% & 30,04 & 93,86 \% \\ \text { Matemática } & 33,91 & 94,66 \% & 34,07 & 94,90 \% & 33,99 & 94,78 \% \\ \text { y Física } & 32,06 & 91,18 \% & 32,09 & 91,25 \% & 32,08 & 91,22 \% \\ \text { Parvularia } & 32,79 & 94,13 \% & 32,80 & 94,15 \% & 32,80 & 94,14 \% \\ \text { Plurilingüe } & 31,69 & 92,44 \% & 31,69 & 92,45 \% & 31,69 & 92,44 \% \\ \text { Psicología Educativa } & \text { PROMEDIOS FFLCE } & & & & & \end{array}$

Cuadro 6. Promedio de asistencia y calificaciones según la variable sexo

Según los resultados observados en el Cuadro 6, no se evidencia diferencia alguna en el rendimiento académico, comparando a estudiantes hombres y mujeres, su promedio es de 31,69 puntos para los dos grupos. En relación a la asistencia a clases en las asignaturas por estudiantes hombres y mujeres la diferencia es mínima, como consta en el Cuadro 6; por tanto, se puede establecer que no hay diferencias significativas en los promedios de calificaciones y promedio de asistencias entre estudiantes hombres y mujeres.

\begin{tabular}{|c|c|c|c|c|}
\hline $\begin{array}{l}\text { Grupos } \\
\text { de carreras }\end{array}$ & $\begin{array}{l}\text { Asignaturas } \\
\text { Periodo } 2016 \text { - } 2017\end{array}$ & $\begin{array}{l}\text { Puntajes } \\
\text { altos } \\
\text { sobre } 40 \\
\end{array}$ & $\begin{array}{l}\text { Asignaturas } \\
\text { Periodo } \\
2016-2017 \\
\end{array}$ & $\begin{array}{l}\text { Puntajes } \\
\text { bajos sobre } \\
40\end{array}$ \\
\hline \multirow{3}{*}{$\begin{array}{l}\text { Ciencias del } \\
\text { Lenguaje y } \\
\text { Literatura }\end{array}$} & $\begin{array}{r}\text { Realidad Cultural y } \\
\text { Ecológica }\end{array}$ & 38,08 & $\begin{array}{r}\text { Didáctica de Lengua } \\
\text { y Literatura I }\end{array}$ & 26,16 \\
\hline & Pedagogía & 37,00 & $\begin{array}{r}\text { Didáctica de Lengua } \\
\text { y Literatura II }\end{array}$ & 27,47 \\
\hline & Literatura Universal V & 36,98 & Investigación III & 28,20 \\
\hline \multirow{3}{*}{$\begin{array}{l}\text { Ciencias } \\
\text { Naturales }\end{array}$} & $\begin{array}{r}\text { Pasantía y Trabajo de } \\
\text { Grado }\end{array}$ & 39,88 & Física & 26,89 \\
\hline & Practica Preprofesional IIII & 39,75 & Química General I & 27,04 \\
\hline & Práctica Preprofesional II & 38,72 & Química General II & 28,86 \\
\hline \multirow{3}{*}{$\begin{array}{l}\text { Ciencias } \\
\text { Sociales }\end{array}$} & Práctica Preprofesional I & 40,00 & Filosofía II & 27,38 \\
\hline & Práctica Preprofesional IV & 39,29 & Historia Del Ecuador & 27,47 \\
\hline & Práctica Preprofesional III & 39,14 & Investigación I & 27,68 \\
\hline \multirow{3}{*}{$\begin{array}{l}\text { Comercio y } \\
\text { Administra } \\
\text { ción }\end{array}$} & Legislación Educativa & 37,55 & $\begin{array}{r}\text { Filosofía de la } \\
\text { Educación }\end{array}$ & 0,00 \\
\hline & Practica Preprofesional V & 36,22 & $\begin{array}{l}\text { Metodología de } \\
\text { Investigación I }\end{array}$ & 0,00 \\
\hline & Práctica Preprofesional III & 35,46 & Estadística I & 5,50 \\
\hline \multirow{3}{*}{ Informática } & Deontología Profesional & 38,80 & Física I & 19,18 \\
\hline & Electrónica & 38,04 & Matemática III & 21,83 \\
\hline & Trabajo de Grado & 37,25 & Matemática I & 21,83 \\
\hline Inglés & Tic I & 37,03 & $\begin{array}{r}\text { Lingüística } \\
\text { Contrastiva } \mathrm{V}\end{array}$ & 25,44 \\
\hline
\end{tabular}




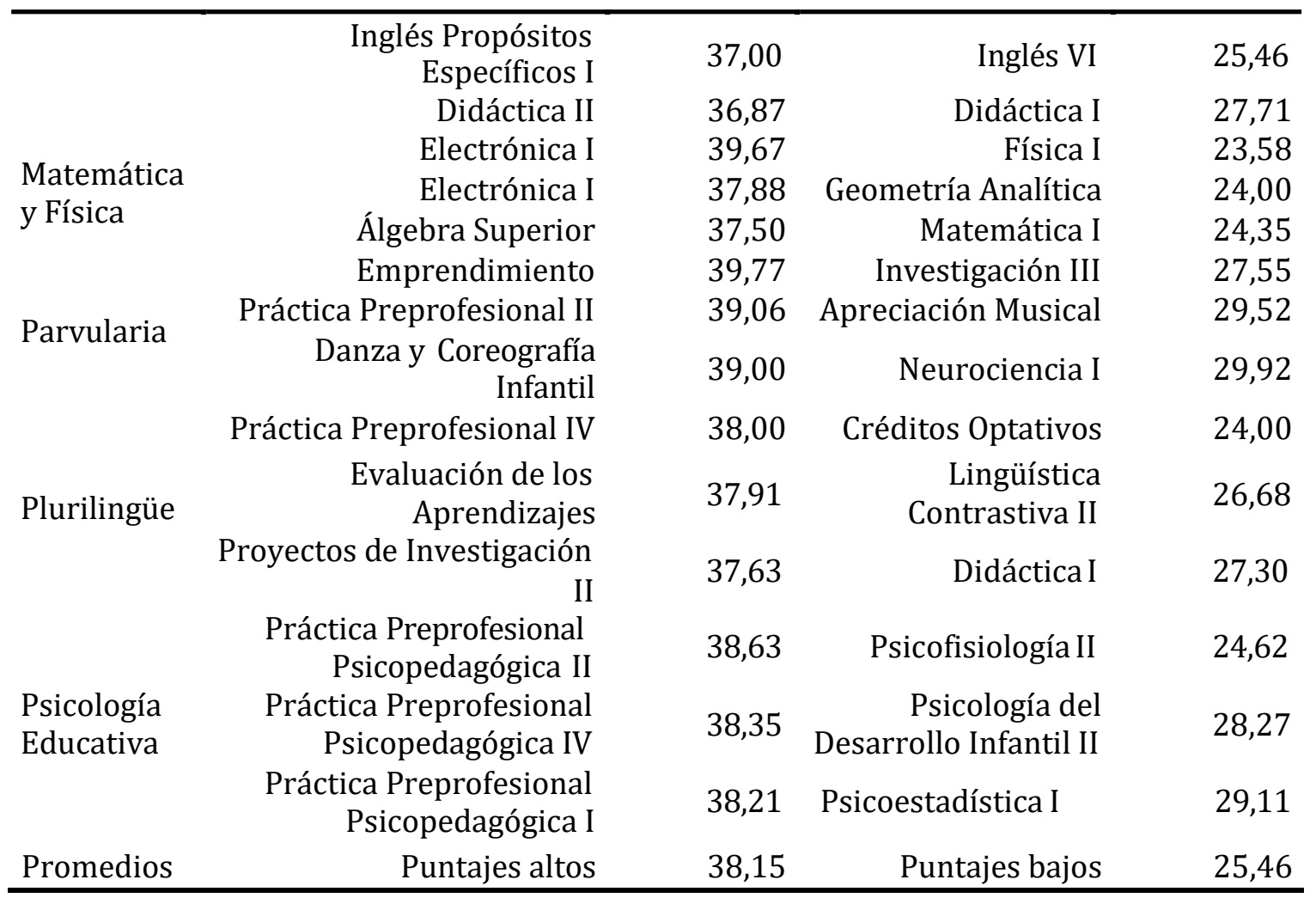

Cuadro 7. Notas más altas y más bajas en asignaturas por carrera de la FFLCE

Asignaturas con altos y bajos promedios de calificaciones. En el Cuadro 7, se presenta por Carreras las tres asignaturas con mayor y con menor promedio de rendimiento académico del estudiantado de la Facultad deFilosofía.

Se evidencia que, en la asignatura de Práctica Preprofesional las calificaciones estudiantiles son más altas, muy cercanas a los 40/40 puntos; mientras que en asignaturas de especialidad presentan los promedios más bajos. Las tres asignaturas por Carrera con los puntajes más altos se encuentran en los intervalos de 36,22 a 40 puntos y que presentan una media aritmética de 38,15 / 40 puntos. Mientras que los puntajes más bajos por grupos de tres asignaturas por Carrera se calculó de aquellas ubicadas en el rango de 29,92 hacia abajo, resultando una media aritmética de 25,46 / 40 puntos.

La asignatura de Práctica preprofesional I de la Carrera de Ciencias Sociales presenta el promedio de calificación más alto de todas las Carreras con 40 / 40 puntos. Mientras que la asignatura de Estadística I de Comercio y Administración tienen el promedio más bajo entre las 10 Carreras con un promedio de 5,5 / 40 puntos. Llama la atención que las asignaturas Filosofía de la Educación y Metodología de Investigación I de la Carrera de Comercio y Administración constan con promedios de notas de 0 puntos como consta en el Cuadro 7.

\section{Conclusiones}

El rendimiento académico expresado en las calificaciones logradas por el estudiantado de las diez carreras de la Facultad de Filosofía, Letras y Ciencias de la Educación de la Universidad Central del Ecuador, en el período académico 2016-2017 es en promedio bueno; es decir; se cumplieron los objetivos de promoción estudiantil con satisfacción, sin

\section{Licencia Creative Commons Atribución 4.0 Internacional (CC BY 4.0)}

Revista Cátedra, 2(1), pp. 44-59, enero-abril 2019. e-ISSN: 2631-2875

https://doi.org/10.29166/catedra.v2i1.1552 
embargo, es de nivel intermedio en la tabla de escalas de los Lineamientos para Evaluación Estudiantil de Grado de la UCE. Las carreras ubicadas en los extremos superior e inferior de la media de rendimiento institucional son Parvularia e Informática respectivamente.

La asistencia estudiantil a clases está en un rango de porcentaje muy bueno, bastante por arriba del estándar mínimo regulado por la Universidad Central del Ecuador, lo que implica que hay responsabilidad estudiantil por sus clases presenciales y control docente en el aula. Las carreras de Parvularia e Inglés destacan positivamente en la calidad de asistencia, mientras que la Carrera de Comercio es observada como aquella de más baja asistencia estudiantil. La variable sexo no es por sí misma factor incidente en el rendimiento académico estudiantil de la Facultad de Filosofía. Los promedios de rendimiento académico $\mathrm{y}$ asistencias a clases no presentan diferencias significativas entre estudiantes hombres y mujeres.

La correlación entre rendimiento académico y asistencia estudiantil calculada con el coeficiente de correlación de Pearson, es de 0,75, que significa que hay una correlación positiva moderada y directamente proporcional entre las variables en estudio. Lo que implica que si se incrementa el porcentaje de asistencia a clases por parte del estudiante, también su rendimiento académico sube.

En la Facultad, el promedio de aprobaciones en el semestre estudiado es muy alto, superior al 90\%, incluyendo rendimiento académico y asistencia como requisitos obligatorios. Sin embargo, hay tres carreras que se manifiestan con mayor porcentaje de repitencia: Informática, Matemática y Física, y Comercio y Administración. En tanto Parvularia, Ciencias del Lenguaje y Psicología tienen menor porcentaje de estudiantes reprobados.

En cuanto a las asignaturas con mayor y menor rendimiento académico estudiantil, destaca Práctica Preprofesional y un grupo importante de asignaturas con un rendimiento académico excelente, muy cercano al estándar máximo de promoción. Son recurrentes también los promedios bajos en asignaturas de especialidad en todas las carreras estudiadas, siendo más evidente este fenómeno en la carrera de Comercio y Administración.

Del estudio comparativo entre rendimiento académico y asistencia por sexo se comprueba fundamentalmente similitud en los puntajes promedio entre estudiantes hombres y mujeres de las diez carreras, con una mínima diferencia en la asistencia a clases favorable a las estudiantes mujeres. Para sostener el estudio fue necesario evaluar la carga de asignaturas por carrera en el contexto general de Facultad, considerando aquellas de formación profesional docente y las de formación especializada de las Carreras, siendo Inglés la Carrera con un menor número de asignaturas (41), mientras que Parvularia tiene el mayor número de asignaturas (69). La media aritmética de asignaturas por carrera $(56,5)$ es muy cercana al mandato del Reglamento de Régimen Académico del CES.

Licencia Creative Commons Atribución 4.0 Internacional (CC BY 4.0)

Revista Cátedra, 2(1), pp. 44-59, enero-abril 2019. e-ISSN: 2631-2875

https://doi.org/10.29166/catedra.v2i1.1552 


\section{Bibliografía}

Arribas, J. M. (2014). Valoración del rendimiento académico de los alumnos de la Facultad de Educación de la UVA (Segovia) en el primer año de implantación de grado. Revista de Educación a Distancia, 1(43), 1-22

Carretero, M. (2009). Constructivismo y Educación. Buenos Aires, Argentina: Paidos

Consejo de Educación Superior. (2016). Reglamento del Sistema de Evaluación Estudiantil Obtenido de http://www.uleam.edu.ec/wp-content/uploads/2016/10/

Consejo de Educación Superior. (2017). Reglamento de Régimen Académico. Quito: CES.

Garbanzo, G. M. (2007). Factores asociados al rendimiento académico en estudiantes universitarios, una reflexión desde la calidad de la educación superior pública. Revista Educación. 31(1), 43-63

Murillo, E. (2013). Factores que inciden en el rendimiento académico de matemáticas. San Pedro Sula: Universidad Pedagógica de Honduras.

Navarro, R. E. (2003). El rendimiento académico: concepto, investigación y desarrollo. Revista Electrónica Iberoamericana sobre Calidad, Eficacia y Cambio en Educació n, 1(2), 1-13

Rodríguez, N. (2016). Lineamientos para evaluacion estudiantil de grado. Quito: UCE.

Rodríguez, N. (2017). Lineamientos para la Evaluación Estudiantil de Grado. Quito: UCE Obtenido de https://bit.ly/2xFF6gu

$\begin{array}{llllll}\text { Salvador } & \mathrm{L} & (\mathrm{sf}) \text { Estadística } & \text { Elemental. }\end{array}$ http://personales.unican.es/salvadol/apuntes2b.pdf

Suárez, A. (2011). http://organizandoelcentroeducativo.blogspot.com. Obtenido de https://bit.ly/2xBIda1

Universidad Central del Ecuador. (2016). Estatuto Universitario. Quitio: Universitaria UCE.

Universidad Central del Ecuador. (2017). Instructivo para la Evaluación Estudiantil de Grado. Quito: UCE.

Universidad de las Palmas Gran Canaria. (2011). Definición de asistencia regular y circunstancias objetivas de justificación de ausencia de clase. Obtenido de https://bit.ly/2MXVi26

Universidad de Loyola Andalucía. (s.f., portada). La importancia de la asistencia a clases. obtenido de https://www.etea.com/web/as00510/asistencia seccion

Revista Cátedra, 2(1), pp. 44-59, enero-abril 2019. e-ISSN: 2631-2875

https://doi.org/10.29166/catedra.v2i1.1552 


\section{Autores}

SEGUNDO BARRENO-FREIRE Licenciado en Ciencias de la Educación, Magister en Gerencias de Proyectos Educativos y Sociales, Doctorando de Investigación Educativa en la Universidad de Alicante.

Docente investigador de la Carrera de Pedagogía de las Ciencias Experimentales Informática; Profesor y Asesor de tesis del Instituto de Posgrado de la Facultad de Filosofía, Letras y Ciencias de la Educación de la Universidad Central del Ecuador.

OSWALDO FABIÁN HARO-JÁCOME Doctor en Investigación Socioeducativa, Magister en Educación, Licenciado en Filosofía y Ciencias Socioeconómicas, Doctorando de Investigación Educativa en la Universidad de Alicante.

Docente investigador de la Carrera de Pedagogía de la Historia y las Ciencias Sociales; Director del Instituto Superior de Extensión Universitaria; Director de la Carrera Plurilingüe; Docente de la Universidad de las Fuerzas Armadas ESPE.

PAOLA FERNANDA FLORES-YANDÚN Licenciado en Ciencias de la Educación, Magister en Diseño y Gestión de Proyectos Socio Educativos.

Docente de Metodología de Investigación de la Universidad Central del Ecuador. Docente investigador, Tutora de Tesis, gestora de Titulación y Coordinadora Académica de las Carrera de Administración Educativa, Informática Educativa, Educación Primaria de la Universidad de Guayaquil. Trabajos de consultoría para el Instituto de Altos Estudios Nacionales IAEN. 